\title{
Inverse Scattering for the Heat Operator and Evolutions in $2+1$ Variables
}

\author{
Mladen Victor Wickerhauser
}

Department of Mathematics, The University of Georgia, Athens, Georgia 30602, USA

\begin{abstract}
The asymptotic behavior of functions in the kernel of the perturbed heat operator $\partial_{1}^{2}-\partial_{2}-u(x)$ suffice to determine $u(x)$. An explicit formula is derived using the $\bar{\partial}$ method of inverse scattering, complete with estimates for small and moderately regular potentials $u$. If $u$ evolves so as to satisfy the Kadomtsev-Petviashvili (KP II) equation, the asymptotic data evolve linearly and boundedly. Thus the KP II equation has solutions bounded for all time. A method for calculating nonlinear evolutions related to KP II is presented. The related evolutions include the so-called "KP II Hierarchy" and many others.
\end{abstract}

\section{Introduction}

Let $u=u(x)$ denote both a function of $x \in \mathbb{R}^{2}$ and the operation of multiplication by that function. This work investigates the inverse scattering problem for the differential operator

$$
\partial_{1}^{2}-\partial_{2}-u
$$

There are two main results. First, for small smooth $u \in L^{1} \cap L^{2}\left(\mathbb{R}^{2}\right)$, the operator is determined by the leading coefficients of asymptotically exponential functions in its kernel. That theorem has several parts. Let $z \in \mathbb{C}$, write $v=v(z)=\left(z, z^{2}\right) \in \mathbb{C}^{2}$, and let $m(x, z)$ be a bounded function such that $\psi=e^{x \cdot v} m(x, z)$ is in the kernel of (I.1). Then for $u$ small in $L^{1} \cap L^{2}\left(\mathbb{R}^{2}\right)$ there is a unique such $m$ satisfying $m(x, z) \rightarrow 1$ as $|x| \rightarrow \infty$ (Theorem 1.II). Hence $\psi$ is asymptotic to $e^{x \cdot v}$. If $u$ has some decay as $|x| \rightarrow \infty$, then $m$ has the asymptotic behavior

$$
m=1+\frac{\beta(z)}{x_{1}+2 z x_{2}}+\frac{\alpha(z) e^{x(\bar{v}-v)}}{x_{1}+2 \bar{z} x_{2}}+o\left(\frac{1}{|x|}\right) \text { as }|x| \rightarrow \infty \quad \text { for } \operatorname{Im} z \neq 0 .
$$

Here $\alpha$ and $\beta$ are bounded functions, with

$$
\alpha(z)=\frac{1}{2 \pi i} \operatorname{sgn}(\operatorname{Im} z) \int_{\mathbb{R}^{2}} e^{x \cdot(v-\tilde{v})} u(x) m(x, z) d x
$$

(Theorem 4.II). 
The coefficient $\alpha(z)$ suffices to determine the $\bar{\partial}$ data of the operator, namely the relationship

$$
\bar{\partial} m(x, z)=\alpha(z) e^{x \cdot(\bar{v}-v)} m(x, \bar{z}) \equiv T m
$$

(Proposition 1.III). Finally, if $u$ is sufficiently smooth, the scattering data $\alpha(z)$ has enough decay to guarantee a unique bounded solution $m$ to the $\bar{\partial}$ problem (I.4) with $m(x, z) \rightarrow 1$ as $|\operatorname{Im} z| \rightarrow \infty$ (Theorem 9.III). Hence both $m$ and Tm are determined by the asymptotic coefficient $\alpha(z)$. But then $u$ is determined by:

$$
u(x)=\frac{1}{\pi i} \partial_{1} \int_{\mathbb{C}} e^{x \cdot(\bar{v}-v)} \alpha(z) m(x, \bar{z}) d \bar{z} \wedge d z
$$

(Theorem 3.IV). The maps $u \rightarrow \alpha$ and $\alpha \rightarrow u$ might be called the forward and inverse scattering transforms, respectively. They behave much like the Fourier transform and its inverse. If $u(x)$ has $n$ derivatives in $L^{1} \cap L^{2}\left(\mathbb{R}^{2}\right)$, then $\alpha(z)$ decays like $(1+|v(z)|)^{-n}$ as $|z| \rightarrow \infty$ (Theorem 4.III). Likewise, if $\alpha(z)$ decays like $(1+|v(z)|)^{-n-2}$, then $u(x)$ has $n$ derivatives in $L^{2}\left(\mathbb{R}^{2}\right)$ with bounded Fourier transform. (Theorem 9.IV).

The second main result is a functional calculus for evolutions of $u$. Let $t \in \mathbb{R}^{+}$be thought of as time, let $\dot{f}$ denote $d f / d t$, and introduce a time dependence for $u(x)$ by the Kadomtsev-Petviashvili equation (KP II)

$$
\partial_{1}\left(\dot{u}+\partial_{1}^{3} u+u \partial_{1} u\right)=-\partial_{2}^{2} u \text {. }
$$

Then $\dot{\alpha}(z)=\left(\bar{z}^{3}-z^{3}\right) \alpha(z)$, or $\alpha(z, t)=\alpha(z, 0) \exp t\left[\bar{z}^{3}-z^{3}\right]$. Likewise, if $u$ evolves by the $n^{\text {th }}$ equation of the KP II hierarchy, then $\dot{\alpha}(z)=\left(\bar{z}^{n}-z^{n}\right) \alpha(z)$. These well-known results are contained in Lemma V.I. Since $\bar{z}^{n}-z^{n}$ is purely imaginary, one has $|\alpha(z, t)|=|\alpha(z, 0)|$ for all $z \in \mathbb{C}, t \geqq 0$. By the first main result, there is a solution $u(x, t)$ for all time to the initial value problem for the $n^{\text {th }}$ KP II evolution, if the initial value $u(x, 0)$ is sufficiently small and regular in $L^{1} \cap L^{2}\left(\mathbb{R}^{2}\right)$ (Theorem V.3).

Conversely, suppose $\dot{\alpha}($ i.e., $\dot{T})$ is known. Then $\dot{u}$ is given by Lemma V.2.:

$$
\dot{u}=\frac{1}{\pi i} \partial_{1} \int_{\mathbb{C}} \tilde{m} \dot{T} m
$$

where $\tilde{m}(x, z)$ solves the transpose of the equation solved by $m$. In particular, if $\phi(z) \in C_{0}^{\infty}(\mathbb{C})$, and $\dot{\alpha}(z)=(\phi(\bar{z})-\phi(z)) \alpha(z)$ so that $\dot{T}=[T, \phi]$, then $\dot{u}(x)=$ $1 / \pi i \partial_{1} \int_{\widetilde{C}}\left(\partial_{\bar{z}} \phi(z)\right) m(x, z) \tilde{m}(x, z) d \bar{z} \wedge d z$ gives the evolution of $u$. By the first main result, its initial value problem for small, regular initial data has a solution at least for small $t>0$.

Extending this functional to $\phi(z)=(s-z)^{-1}$ yields the $n^{\text {th }}$ evolution equation in the KP II hierarchy as the coefficient of $s^{-n}$ in the expansion of $\partial_{1}(m(x, s) \tilde{m}(x, s))$ about $s=\infty$. Hence $\dot{T}=\left[T,(s-z)^{-1}\right]$ might be said to generate the KP II hierarchy. Likewise, $\phi(z)=z^{-1}$ yields the nonlinear system

$$
\begin{array}{llll}
\dot{u} & =\partial_{1}(m \tilde{m}), \\
0 & =\left(\partial_{1}^{2}-\partial_{2}-u\right) m, \quad m(x) \rightarrow 1 & \text { as } & |x| \rightarrow \infty, \\
0 & =\left(\partial_{1}^{2}+\partial_{2}-u\right) \tilde{m}, \quad \tilde{m}(x) \rightarrow 1 & \text { as } & |x| \rightarrow \infty .
\end{array}
$$


These are two concisely written examples of the large class of evolutions of $u$ related to linear evolutions of $\alpha$.

The inverse scattering method for solving evolution equations is explained in the classic article [AKNS]. The $\bar{\partial}$ method is introduced in $[\mathrm{BC}]$ and $[\mathrm{BC} 2]$. Formal solutions to the KP equation may be found in $[\mathrm{FA}]$, $[\mathrm{ABF}]$.

The KP hierarchy is defined in [DJKM] and [F], where it is studied for algebraic reasons. A rather impenetrable functional calculus for another scattering problem appears in $[\mathrm{K}]$.

\section{Asymptotically Exponential Solutions to the Perturbed Heat Equation}

Consider the heat operator in 2 variables $x=\left(x_{1}, x_{2}\right)$, perturbed by a multiplicative potential $u(x)$, and the equation for its kernel:

$$
\left(\partial_{1}^{2}-\partial_{2}-u\right) \psi=0
$$

One class of solutions to this equation may be specified by requiring that $\psi$ be asymptotic to a purely exponential solution to the unperturbed equation. More precisely, consider the class of solutions $\psi_{z}(x), z \in \mathbb{C}$, satisfying the conditions:

$$
\psi_{z}(x)=e^{x \cdot v} m(x, z) \quad \text { for } \quad v=v(z)=\left(z, z^{2}\right) ; \quad \lim _{|x| \rightarrow \infty} m(x, z)=1 \text { for each } z .
$$

This class contains the kind of solutions one would expect if the perturbation function $u(x)$ were compactly supported. It is completely determined by the function $m(x, z)$ on the variety $V=\left\{v(z)=\left(z, z^{2}\right) \in \mathbb{C}^{2} \mid z \in \mathbb{C}\right\}$. This function satisfies a boundary value problem in $L^{\infty}\left(\mathbb{R}^{2} \times \mathbb{C}\right)$ :

$$
\begin{aligned}
& {\left[\left(\partial_{1}+z\right)^{2}-\left(\partial_{2}+z^{2}\right)-u(x)\right] m(x, z)=0 .} \\
& \text { For each } \quad z \in \mathbb{C}, \lim _{|x| \rightarrow \infty} m(x, z)=1 .
\end{aligned}
$$

Knowledge of $m$ suffices to determine $u$, but first it is important to know that there is a unique $m$ for a given $u$. Standard methods may be applied. Denote by $\hat{f}$ the Fourier transform of $f$, in the distributional sense, which is chosen to agree on the Schwartz class with the integral

$$
\hat{f}(\xi)=\int_{\mathbb{R}^{2}} \exp [-2 \pi i x \cdot \xi] f(x) d x, \quad \text { where } \quad \xi=\left(\xi_{1}, \xi_{2}\right) \in \mathbb{R}^{2}, \quad \text { and } \quad d x=d x_{1} d x_{2} .
$$

This choice ensures that $f \rightarrow \hat{f}$ is an isometry of $L^{2}\left(\mathbb{R}^{2}\right)$ and has norm 1 as a map from $L^{1}\left(\mathbb{R}^{2}\right)$ to $L^{\infty}\left(\mathbb{R}^{2}\right)$. For functions of more variables, $\hat{f}$ will denote the partial Fourier transform in the first two real variables, with the other variables considered parameters. Denote by $f$ the inverse Fourier transform of $f: f(\xi)=\breve{f}(-\xi)$. Applying the Fourier transform to Eq. (II.3) yields:

$$
\left[\left(2 \pi i \xi_{1}+z\right)^{2}-\left(2 \pi i \xi_{2}+z^{2}\right)\right] \hat{m}(\xi, z)=[u m] \hat{(\xi, z) . ~}
$$

It is convenient to introduce some notation:

$$
P(\xi)=\xi_{1}^{2}-\xi_{2}, \quad \text { so that } \quad V=\left\{v \in \mathbb{C}^{2} \mid P(v)=0\right\}, \quad P_{z}(\xi)=P(2 \pi i \xi+v(z)) .
$$


For each $z \in \mathbb{C}$, there are at most two distinct roots $\xi$ of $P_{z}(\xi)$, namely 0 and a root $\xi_{0}(z)=\operatorname{Im} v(z) / 2 \pi$ which may be parametrized by one complex variable as:

$$
\xi_{0}(z) \equiv\left(-\frac{\operatorname{Im} z}{\pi}, \frac{-2 \operatorname{Re} z \operatorname{Im} z}{\pi}\right)=\left(\frac{\bar{z}-z}{2 \pi i}, \frac{\bar{z}^{2}-z^{2}}{2 \pi i}\right) .
$$

It is now possible to prove a basic existence theorem for the perturbed heat operator:

Theorem 1.II. Suppose $u(x)$ belongs to both $L^{2}\left(\mathbb{R}^{2}\right)$ and $L^{1}\left(\mathbb{R}^{2}\right)$ and is small in the sense that $\|u\|_{L^{1}}+\|u\|_{L^{2}}<\varepsilon$. Then there is a unique solution $m(x, z) \in L^{\infty}\left(\mathbb{R}^{2} \times \mathbb{C}\right)$ to the boundary value problem (II.3).

Proof. Existence follows from the contraction mapping theorem and a technical lemma. Since the constant function 1 is annihilated by $P(\partial+v)$, it is possible to rewrite Eq. (II.5) as a Fredholm integral equation:

$$
[m-1]=\frac{[u(m-1) \hat{]}}{P_{z}}+\frac{\hat{u}}{P_{z}} .
$$

To study this map $\hat{f} \rightarrow\left([u f] / P_{z}\right)$ requires technology:

Lemma 2.II. For each $z \in \mathbb{C}$ there is a subset $E_{z} \subset \mathbb{R}^{2}$ and a constant $C_{z}$ such that

$$
\left\|\frac{1}{P_{z}}\right\|_{L^{1}\left(E_{z}\right)}<C_{z} \text { and }\left\|\frac{1}{P_{z}}\right\|_{L^{2}\left(E_{z}^{c}\right)}<C_{z} .
$$

Furthermore, the sets $E_{z}$ can be chosen in such a way that $C_{z}$ is a bounded function of $z$, and $C_{z} \rightarrow 0$ as $|\operatorname{Im} z| \rightarrow \infty$.

The proof will be found at the end of this theorem. Note some immediate consequences:

\section{Corollary 3.II.}

(i) If $\hat{u} \in L^{2} \cap L^{\infty}\left(\mathbb{R}^{2}\right)$, then $\left(\hat{u} / P_{z}\right) \in L^{1}\left(\mathbb{R}^{2}\right)$ with norm uniformly bounded in $z$.

(ii) The map $f \rightarrow(\hat{u} * f) / P_{z}$ is bounded from $L^{1}\left(\mathbb{R}^{2}\right)$ to $L^{1}\left(\mathbb{R}^{2}\right)$, uniformly in $z$, and has norm $<C_{z}\left(\|\hat{u}\|_{L^{\infty}}+\|\hat{u}\|_{L^{2}}\right) \leqq C_{z}\left(\|u\|_{L^{1}}+\|u\|_{L^{2}}\right)$.

By the contraction mapping theorem in $L^{1}\left(\mathbb{R}^{2}\right)$, Eq. (II.8) has a unique solution $[m-1]^{\hat{n}}(\xi, z)$ for each $z$. Its inverse Fourier transform $m(x, z)$ solves (II.3).

To show uniqueness, suppose $m(x, z) \in L^{\infty}\left(\mathbb{R}^{2} \times \mathbb{C}\right)$ solves (II.3). Then $\hat{m}$ is a tempered distribution which is the sum of the integrable function $\left([\mathrm{um}] / P_{z}\right)$ and a distribution supported at 0 and $\xi_{0}(z)$. Such distributions can only be Dirac masses and their derivatives, and their inverse Fourier transforms can be explicitly calculated. Comparing behavior as $|x| \rightarrow \infty$, it becomes plain that $[m-1]^{\wedge}$ is the unique integrable function which solves (II.8). This completes the proof of the theorem.

It remains to establish Lemma 2.II.

Proof. Start by transforming $P_{z}(\xi)$ into $\xi_{1}^{2}-(\operatorname{Im} z)^{2}+i \xi_{2}$ by the (almost) isometric change of variable: 


$$
\xi_{1}=\frac{1}{2 \pi}\left[\xi_{1}^{\prime}-\operatorname{Im} z\right], \quad \xi_{2}=\frac{1}{2 \pi}\left[\xi_{2}^{\prime}+2 \xi_{1}^{\prime} \operatorname{Re} z+2 \operatorname{Re} z \operatorname{Im} z\right] .
$$

(The Jacobian is evidently $(1 / 2 \pi)^{2}$.) Observe that for any $\delta, 0 \leqq \delta \leqq 1$, one has the following inequality:

$$
\begin{aligned}
\left|\xi_{1}^{2}-(\operatorname{Im} z)^{2}\right| & \geqq \max \left\{\left|\xi_{1}-\operatorname{Im} z\right|, \quad\left|\xi_{1}+\operatorname{Im} z\right|\right\}^{\delta} \min \left\{\left|\xi_{1}-\operatorname{Im} z\right|, \quad\left|\xi_{1}+\operatorname{Im} z\right|\right\}^{2-\delta} \\
& =\left(\left|\xi_{1}\right|+|\operatorname{Im} z|\right)^{\delta} \min \left\{\left|\xi_{1}-\operatorname{Im} z\right|, \quad\left|\xi_{1}+\operatorname{Im} z\right|\right\}^{2-\delta} \\
& \geqq|\operatorname{Im} z|^{\delta} \min \left\{\left|\xi_{1}-\operatorname{Im} z\right|, \quad\left|\xi_{1}+\operatorname{Im} z\right|\right\}^{2-\delta} .
\end{aligned}
$$

This leads to the crude estimate:

$$
\left|\frac{1}{P_{z}(\xi)}\right| \leqq \frac{c}{|\operatorname{Im} z|^{\delta}\left|\xi_{1}-\operatorname{Im} z\right|^{2-\delta}+\left|\xi_{2}\right|}+\frac{c}{|\operatorname{Im} z|^{\delta}\left|\xi_{1}+\operatorname{Im} z\right|^{2-\delta}+\left|\xi_{2}\right|} .
$$

Let $E=\xi \in \mathbb{R}^{2}:\left|\xi_{1}\right|<1,\left|\xi_{2}\right|<1$ and let $E_{z}=[E+(\operatorname{Im} z, 0)] \cup[E-(\operatorname{Im} z, 0)]$. By translation, it is clear that the integrability of $1 / P_{z}(\xi)$ on $E_{z}$ and $E_{z}^{c}$ is equivalent to the integrability on $E$ and $E^{c}$ of $1 /\left(|\operatorname{Im} z|^{\delta}\left|\xi_{1}\right|^{2-\delta}+\left|\xi_{2}\right|\right)$.

Choosing $\delta=0$, an application of the calculus shows that $\left\|1 / P_{z}(\xi)\right\|_{L^{1}\left(E_{z}\right)}$ and $\left\|1 / P_{z}(\xi)\right\|_{L^{2}\left(E_{z}^{c}\right)}$ are both uniformly bounded functions of $z$.

Choosing $\delta=1 / 2$, observe that $\left(|\operatorname{Im} z|^{1 / 2}\left|\xi_{1}\right|^{3 / 2}+\left|\xi_{2}\right|\right)^{-1} \rightarrow 0$ pointwise almost everywhere as $|\operatorname{Im} z| \rightarrow \infty$. By calculus and the dominated convergence theorem, $\left\|1 /\left(|\operatorname{Im} z|^{1 / 2}\left|\xi_{1}\right|^{3 / 2}+\left|\xi_{2}\right|\right)\right\|_{L^{1}(E)} \rightarrow 0$ and $\left\|1 /\left(|\operatorname{Im} z|^{1 / 2}\left|\xi_{1}\right|^{3 / 2}+\left|\xi_{2}\right|\right)\right\|_{L^{2}\left(E^{c}\right)} \rightarrow 0$ as $|\operatorname{Im} z| \rightarrow \infty$. But then the same result holds for $1 / P_{z}(\xi)$ when $E$ is replaced by $E_{z}$.

Suppose that $u(x)$ is small in both $L^{1}\left(\mathbb{R}^{2}\right)$ and $L^{2}\left(\mathbb{R}^{2}\right)$, so that a unique solution $m(x, z)$ exists by Theorem 1.II. The notion of "scattering data" suggests a function, depending on the energy parameter $z$, which can be determined by a knowledge of $m(x, z)$ at points "far away" from the potential. Of course, this makes sense only if $u$ is compactly supported, but it is possible to generalize this notion of "far away" by using asymptotic expansions.

Consider as candidates for "scattering data" the coefficients of the leading terms of the asymptotic expansion for $m(x, z)$ as $|x| \rightarrow \infty$.

It remains to calculate this expansion. One can use the integral equation:

$$
m(x, z)=1+\int_{\mathbb{R}^{2}} \exp [2 \pi i x \cdot \xi] \frac{[u m] \hat{(\xi, z)}}{P_{z}(\xi)} d \xi .
$$

Since $\left([u m] \hat{/} P_{z}\right) \in L^{1}\left(\mathbb{R}^{2}\right)$, the Riemann-Lebesgue lemma implies that the integral tends to 0 as $|x| \rightarrow \infty$. However, those places where $[u m] / P_{z}$ is discontinuous, namely the roots of $P_{z}$, should contribute the slowest decay. This principle gives rise to the following result:

Theorem 4.II. Suppose $m(x, z)$ is a solution to Eq. (II.9) in $L^{\infty}\left(\mathbb{R}^{2} \times \mathbb{C}\right)$, and that

$$
(1+|x|)|u(x)| \in L^{1}\left(\mathbb{R}^{2}\right) \cap L^{2}\left(\mathbb{R}^{2}\right) .
$$

Then if $\operatorname{Im} z \neq 0, m$ has the asymptotic behavior

$$
m(x, z)=1+\frac{\beta(z)}{x_{1}+2 z x_{2}}+\frac{\alpha(z) e^{2 \pi i x \cdot \xi_{0}(z)}}{x_{1}+2 \bar{z} x_{2}}+o\left(\frac{1}{|x|}\right) \text { as }|x| \rightarrow \infty .
$$


Here

$$
\alpha(z)=\frac{1}{2 \pi i}[u m] \hat{(}\left(\xi_{0}(z), z\right) \operatorname{sgn}(\operatorname{Im} z), \quad \beta(z)=\frac{1}{2 \pi i}[u m] \hat{]}(0, z) \operatorname{sgn}(\operatorname{Im} z)
$$

Proof. Fix $z \in \mathbb{C}$ with $\operatorname{Im} z \neq 0$. Write $1=\phi_{0}(\xi)+\phi_{1}(\xi)+\phi_{\infty}(\xi)$, a smooth nonnegative partition of unity, where $\phi_{0}=1$ in a neighborhood of $0, \phi_{1}=1$ in a neighborhood of $\xi_{0}(z)=\left((\bar{z}-z) / 2 \pi i,\left(\bar{z}^{2}-z^{2}\right) / 2 \pi i\right)$, and $\phi_{\infty}=1$ in a neighborhood of $\infty$. Observe that $\phi_{0}$ and $\phi_{1}$ are compactly supported, and $\phi_{\infty}$ is supported away from the zeros of $P_{z}$. Then, since $m(x, z)$ is a solution of Eq. (II.9), the following is an equation in $L^{1}\left(\mathbb{R}^{2}\right)$ :

$$
[m-1]=\frac{[u m] \hat{]}}{P_{z}}=\frac{[u m]}{P_{z}} \phi_{0}+\frac{[u m] \hat{]}}{P_{z}} \phi_{1}+\frac{[u m]}{P_{z}} \phi_{\infty} .
$$

Thus

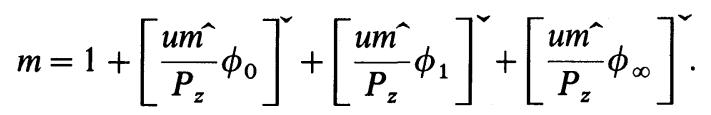

Each of these terms may be estimated in turn. To do this, introduce the derivations

$$
\begin{aligned}
& \partial \equiv \frac{1}{2 \pi i}\left(\frac{\partial}{\partial \xi_{1}}+2 z \frac{\partial}{\partial \xi_{2}}\right)=\left(x_{1}+2 z x_{2}\right) \hat{)} \\
& \bar{\partial} \equiv \frac{1}{2 \pi i}\left(\frac{\partial}{\partial \xi_{1}}+2 \bar{z} \frac{\partial}{\partial \xi_{2}}\right)=\left(x_{1}+2 \bar{z} x_{2}\right) \hat{.}
\end{aligned}
$$

Case $\infty$. By the decay condition on $u,[u m]$ has at least one derivative in $L^{2} \cap L^{\infty}$. A simple calculation shows that $\partial\left(\phi_{\infty} / P_{z}\right)$ belongs to $L^{2}$. Thus $\partial\left(\left([u m] \hat{P} / P_{z}\right) \phi_{\infty}\right)=(\partial[u m] \hat{)})\left(\phi_{\infty} / P_{z}\right)+[u m] \hat{\partial}\left(\left(\phi_{\infty} / P_{z}\right)\right)$ belongs to $L^{1}$, and by the Riemann-Lebesgue lemma, $\left(\left([u m] / P_{z}\right) \phi_{\infty}\right)^{\iota}=o(1 /|x|)$ as $|x| \rightarrow \infty$.

Case 0 . Since $\operatorname{Im} z \neq 0,1 /\left(x_{1}+2 z x_{2}\right)$ is smooth and bounded in the set $|x|>1$. There,

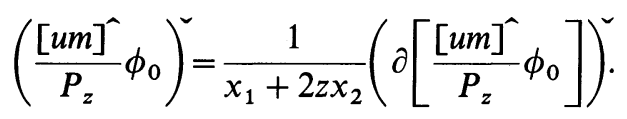

Since $\partial$ is a derivation and [um] has one derivative in $L^{2} \cap L^{\infty}$, another application of the Riemann-Lebesgue lemma shows

$$
\left(\frac{[u m]}{P_{z}} \phi_{0}\right)^{\smile}=\frac{1}{x_{1}+2 z x_{2}}\left([u m] \hat{\partial}\left(\frac{\phi_{0}}{P_{z}}\right)\right)^{\smile}+o\left(\frac{1}{|x|}\right) .
$$

Now $\partial\left(\phi_{0} / P_{z}\right)$ is a distribution which may be computed by standard regularization methods. It differs from an integrable function by the compactly supported singular part

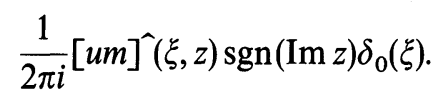


Recognizing $\beta(z)$ from its definition, one concludes:

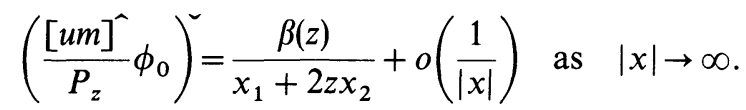

Case 1. As in case 0 , one may write

$$
\left(\frac{[u m]}{P_{z}} \phi_{1}\right)^{\smile}=\frac{1}{x_{1}+2 \bar{z} x_{2}}\left([u m] \tilde{\partial}\left(\frac{\phi_{1}}{P_{z}}\right)\right)^{\smile}+o\left(\frac{1}{|x|}\right) \text { as }|x| \rightarrow \infty .
$$

Here again $\bar{\partial}\left(\phi_{1} / P_{z}\right)$ is a distribution which differs from an integrable function by

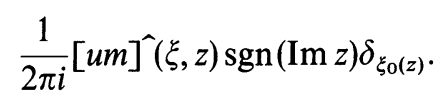

As before, the little $o$ swallows the integrable part, leaving what is recognizable as

$$
\left(\frac{[u m]}{P_{z}} \phi_{1}\right)^{\frown}=\frac{\alpha(z) e^{2 \pi i x \cdot \xi_{0}(z)}}{x_{1}+2 \bar{z} x_{2}}+o\left(\frac{1}{|x|}\right) .
$$

Putting all terms together yields the result. This completes the proof except for the calculation of $\partial\left(\phi_{0} / P_{z}\right)$ and $\bar{\partial}\left(\phi_{1} / P_{z}\right)$, which turns out to be useful later. Observe that both $\phi_{0} / P_{z}$ and $\phi_{1} / P_{z}$ are integrable. Thus by dominated convergence, they may be regularized by multiplication with $r_{\varepsilon}=1-\exp \left[-\left|P_{z}\right|^{2} / \varepsilon^{2}\right]$. Also, the choice of $\partial$ and $\bar{\partial}$ insures that $\lim _{\varepsilon \rightarrow 0} \partial\left(\phi_{0} / P_{z}\right) r_{\varepsilon}$ and $\lim _{\varepsilon \rightarrow 0} \bar{\partial}\left(\phi_{1} / P_{z}\right) r_{\varepsilon}$ both exist in $L^{1}\left(\mathbb{R}^{2}\right)$. Then in the sense of distributions, one can take limits:

$$
\partial\left(\frac{\phi_{0}}{P_{z}}\right)=\text { integrable }+\frac{\phi_{0}}{P_{z}} \lim _{\varepsilon \rightarrow 0} \partial r_{\varepsilon}, \quad \bar{\partial}\left(\frac{\phi_{1}}{P_{z}}\right)=\text { integrable }+\frac{\phi_{1}}{P_{z}} \lim _{\varepsilon \rightarrow 0} \bar{\partial} r_{\varepsilon}
$$

But the rightmost terms are straightforward to calculate:

$$
\begin{aligned}
& \frac{\phi_{0}}{P_{z}} \lim _{\varepsilon \rightarrow 0} \partial r_{\varepsilon}=-4 \pi i \phi_{0}\left[1-\frac{\xi_{1}}{\xi_{1}-\xi_{0}^{1}(z)} \frac{\bar{P}_{z}}{P_{z}}\right]_{\varepsilon \rightarrow 0} \frac{\xi_{1}-\xi_{0}^{1}(z)}{\varepsilon^{2}} \exp \left[-\left|P_{z}\right|^{2} / \varepsilon^{2}\right], \\
& \frac{\phi_{1}}{P_{z}} \lim _{\varepsilon \rightarrow 0} \partial r_{\varepsilon}=-4 \pi i \phi_{1}\left[1-\frac{\xi_{1}-\xi_{0}^{1}(z)}{\xi_{1}} \frac{\bar{P}_{z}}{P_{z}}\right] \lim _{\varepsilon \rightarrow 0} \frac{\xi_{1}}{\varepsilon^{2}} \exp \left[-\left|P_{z}\right|^{2} / \varepsilon^{2}\right] \text {. }
\end{aligned}
$$

At this point, it is useful to single out the evaluation of these limits as a lemma:

Lemma 5.II. In the sense of distributions, as $\varepsilon \rightarrow 0$,

$$
\begin{gathered}
\frac{\xi_{1}}{\varepsilon^{2}} \exp \left[-\left|P_{z}\right|^{2} / \varepsilon^{2}\right] \rightarrow-\frac{1}{8 \pi^{2}} \operatorname{sgn}(\operatorname{Im} z) \delta_{\xi_{0}(z)}, \\
\frac{\xi_{1}-\xi_{0}^{1}(z)}{\varepsilon^{2}} \exp \left[-\left|P_{z}\right|^{2} / \varepsilon^{2}\right] \rightarrow-\frac{1}{8 \pi^{2}} \operatorname{sgn}(\operatorname{Im} z) \delta_{0} .
\end{gathered}
$$

Proof of Lemma 5.II. Equations (II.13) and (II.14) are translates of each other because of the identity:

$$
P_{z}\left(\xi+\xi_{0}(z)\right)=P_{z}(\xi) .
$$


The rest of the calculation is an exercise in change of variable and dominated convergence.

Observe that $\left[1-\left(\bar{P}_{z} / P_{z}\right) \xi_{1} /\left(\xi_{1}-\xi_{0}^{1}(z)\right)\right]$ is continuous at 0 , and that $\left[1-\left(\bar{P}_{z} / P_{z}\right)\left(\xi_{1}-\xi_{0}^{1}(z)\right) / \xi_{1}\right]$ is continuous at $\xi_{0}(z)$. Each is equal to 1 at those points. Applying the lemma to Eq. (II.12) gives

$$
\frac{\phi_{0}}{P_{z}} \lim _{\varepsilon \rightarrow 0} \partial r_{\varepsilon}=\frac{1}{2 \pi i} \operatorname{sgn}(\operatorname{Im} z) \delta_{\xi_{0}}, \quad \frac{\phi_{1}}{P_{z}} \lim \delta r_{\varepsilon}=\frac{1}{2 \pi i} \operatorname{sgn}(\operatorname{Im} z) \delta_{0} .
$$

Using this result in Eq. (II.11) completes the proof of Theorem 4.II.

\section{Calculating the Asymptotic Behavior via $\partial_{\bar{z}}$}

Suppose $\psi(x, z)$ is an asymptotically exponential solution to $P(\partial) \psi=u \psi$. Write

$$
\psi(x, z)=e^{x \cdot v(z)}\left[1+\frac{\beta(z)}{x_{1}+2 z x_{2}}+\frac{\alpha(z) e^{2 \pi i x \cdot \xi_{0}(z)}}{x_{1}+2 \bar{z} x_{2}}+o\left(\frac{1}{|x|}\right)\right] .
$$

It can be shown that if $u(x)$ evolves in time to satisfy the Kadomtsev-Petviashvili equation,

$$
\partial_{1}\left(\dot{u}+u \partial_{1} u+\partial_{1}^{3} u\right)=-\partial_{2}^{2} u,
$$

then $\alpha(z)$ and $\beta(z)$ evolve according to the equations

$$
\dot{\alpha}(z)=\left(\bar{z}^{3}-z^{3}\right) \alpha(z), \quad \dot{\beta}(z)=0 .
$$

These evolutions are trivial to solve: $\alpha(z, t)=\alpha(z, 0) \exp t\left[\bar{z}^{3}-z^{3}\right]$ and $\beta(z, t)=\beta(z, 0)$. Hence it is a matter of great interest to reconstruct the potential $u(x)$ from $\alpha$ and $\beta$, since that would lead to a solution of the KP equation.

An observation of R. Beals and R. R. Coifman $[\mathrm{BC}]$ has shown that the asymptotic data $\alpha(z)$ determines the relationship between $\psi$ and $\partial_{\bar{z}} \psi$. But knowing this relationship amounts to knowing $\psi$ and consequently $u$.

Write $\psi(x, z)=e^{x \cdot v} m(x, z)$ with $m$ bounded. Then $m$ satisfies $P(\partial+v) m=u m$. This is an analytic family of differential equations in the parameter $z \in \mathbb{C}$, where $v=\left(z, z^{2}\right)$. Denote by $\partial_{\bar{z}}$ the derivative with respect to the conjugate of this parameter. Then

$$
P(\partial+v) \partial_{\bar{z}} m=u \partial_{\bar{i}} m .
$$

Using the existence-uniqueness theorem for this equation, one has $\partial_{\bar{z}} m=T m$ where $T$ is some linear operator. Call the map $u \rightarrow T$ the Forward Scattering Transform. It may be calculated as follows:

Proposition 1.III. Suppose $\|u\|_{L^{1}}+\|u\|_{L^{2}}$ is small, so that $m(x, z)$ is the unique solution to

$$
P(\partial+v) m=u m, \quad m \in L^{\infty}\left(\mathbb{R}^{2} \times \mathbb{C}\right), \lim _{|x| \rightarrow \infty} m(x, z)=1 .
$$

Then $\partial_{\bar{z}} m(x, z)$ exists and is bounded on $\mathbb{R}^{2} \times \mathbb{C}$, and in fact may be written as

$$
\partial_{\bar{z}} m(x, z)=\alpha(z) \exp \left[2 \pi i x \cdot \xi_{0}(z)\right] m(x, \bar{z}) \equiv \operatorname{Tm}
$$

with $\alpha(z)=(-1 / 2 \pi i) \operatorname{sgn}(\operatorname{Im} z)[u m]^{\hat{(}}\left(\xi_{0}(z), z\right)$. 
Proof. Write $G m(x, z)=\left([u m]^{\wedge} / P_{z}\right)^{-}=\int_{\mathbb{R}^{2}}\left(e^{2 \pi i x \cdot \xi} / P_{z}(\xi)\right)[u m]^{\wedge}(\xi, z) d \xi$. Then $m=1+$ Gm. By Theorem 1.II, $\|G\|_{L^{\infty} \rightarrow L^{\infty}}$ can be made smaller than one by insisting that $\|u\|_{L^{1}}+\|u\|_{L^{2}}$ be small. Then one can write

$$
m=(I-G)^{-1} 1 .
$$

Hence $\partial_{\bar{z}} m=(I-G)^{-1}\left(\partial_{\bar{z}} G\right) m$. But since $\left([u m]^{\hat{n}} / P_{z}\right)$ is integrable, dominated convergence allows regularizing $G$ and then differentiating under the integral:

$$
\left(\partial_{\bar{z}} G\right) m=\lim _{\varepsilon \rightarrow 0} \int_{\mathbb{R}^{2}} e^{2 \pi i x \cdot \xi} \partial_{\bar{z}}\left(\frac{r_{\varepsilon}}{P_{z}}\right)[u m]^{\hat{\gamma}}(\xi, z) d \xi,
$$

where $r_{\varepsilon}=1-\exp \left[-\left|P_{z}\right|^{2} / \varepsilon^{2}\right]$.

It is straightforward to verify that

$$
\partial_{\bar{z}}\left(r_{\varepsilon} / P_{z}\right)=\frac{-4 \pi i \xi_{1}}{\varepsilon^{2}} \exp \left[-\left|P_{z}\right|^{2} / \varepsilon^{2}\right]
$$

Lemma 5.II. shows that this tends to

$$
\frac{-1}{2 \pi i} \operatorname{sgn}(\operatorname{Im} z) \delta_{\xi_{0}(z)}
$$

Hence $\left(\partial_{\bar{z}} G\right) m(x, z)=\alpha(z) \exp \left[2 \pi i x \cdot \xi_{0}(z)\right]$, where $\alpha(z)$ is defined by the statement of the proposition. Thus $\partial_{\bar{z}} m=\alpha(z)(I-G)^{-1} \exp \left[2 \pi i x \cdot \xi_{0}(z)\right]$, since $G$ commutes with multiplication by functions of $z$ alone. It merely remains to calculate

$$
(I-G)^{-1} \exp \left[2 \pi i x \cdot \xi_{0}(z)\right] .
$$

Call this function $n(x, z)$. It is bounded because the exponential is bounded and $I-G$ is invertible on $L^{\infty}$. It satisfies the equation

$$
P(\partial+v) n=u n, \quad n(x, z) \sim \exp \left[2 \pi i x \cdot \xi_{0}(z)\right] \quad \text { as } \quad|x| \rightarrow \infty .
$$

But then $n^{\prime}=n \exp \left[-2 \pi i x \cdot \xi_{0}(z)\right]$ satisfies

$$
P(\partial+\bar{v}) n^{\prime}=u n^{\prime}, \quad n^{\prime}(x, z) \rightarrow 1 \quad \text { as } \quad|x| \rightarrow \infty .
$$

There is a unique solution to this equation, which has already been named $m(x, \bar{z})$. Putting the parts together yields

$$
\partial_{\bar{z}} m(x, z)=\alpha(z) m(x, \bar{z}) \exp \left[2 \pi i \xi_{0}(z) \cdot x\right] .
$$

One easy consequence is an estimate on $\alpha(z)$ :

Corollary 2.III. If $\|u\|_{L^{1}}+\|u\|_{L^{2}}$ is small, the function $\alpha(z)$ is bounded. More precisely, there is a bounded function $C_{z}$ verifying $\lim _{|\mathrm{I} z| \rightarrow \infty} C_{z}=0$ such that

$$
|\alpha(z)| \leqq \frac{\|u\|_{L^{1}}}{1-C_{z}\left(\|u\|_{L^{1}}+\|u\|_{L^{2}}\right)} .
$$

Proof. $|\alpha(z)|=\left|[u m]^{\hat{n}}\left(\xi_{0}(z), z\right)\right| \leqq\left\|[u m]^{\hat{}}\right\|_{L^{\infty}} \leqq\|u m\|_{L^{1}} \leqq\|u\|_{L^{1}}\|m\|_{L^{\infty}}$ by various famous inequalities. But $m=(I-G)^{-1} 1$ and Corollary 3.II implies that $\|G\|_{L^{\infty} \rightarrow L^{\infty}}$ 
$\leqq C_{z}\left(\|u\|_{L^{1}}+\|u\|_{L^{2}}\right)$, where $C_{z}$ is bounded and tends to 0 as $|\operatorname{Im} z|$ tends to $\infty$. Then $\|m\|_{L^{\infty}} \leqq\left[1-C_{z}\left(\|u\|_{L^{1}}+\|u\|_{L^{2}}\right)\right]^{-1}$ and the corollary is proved.

Although the asymptotic behavior of $m(x, z)$ as $|x| \rightarrow \infty$ involves more than just $\alpha(z)$, the other features turn out to be unnecessary for the recovery of $m$ or $u$. Introduce:

Definition 3.III. The scattering data $\alpha(z)$ associated to a small potential $u(x)$ by the perturbed heat operator is the function defined by

$$
\alpha(z)=\frac{-1}{2 \pi i} \operatorname{sgn}(\operatorname{Im} z)[u m]\left(\xi_{0}(z), z\right) \quad z \in \mathbb{C} .
$$

Abusing notation, the continuous linear map $T: L^{\infty} \rightarrow L^{\infty}$ determined by $\alpha(z)$ shall also be called scattering data. Here

$$
\operatorname{Tm}(x, z)=\alpha(z) m(x, \bar{z}) \exp \left[2 \pi i x \cdot \xi_{0}(z)\right] .
$$

This abuse of notation is legitimized by the observation that this operator $T$, which agrees with $\partial_{i}$ on asymptotically exponential solutions of the perturbed heat equation, is essentially multiplication by the leading asymptotic behavior of $m$.

The function $\alpha(z)$ behaves much like the Fourier transform of $u$. In particular, if $u$ is smooth then $\alpha$ has rapid decay in some directions. More precisely:

Theorem 4.III. Suppose that $\|u\|_{L^{1}}+\|u\|_{L^{2}}$ is small and that for some multi-index $\gamma=\left(\gamma_{1}, \gamma_{2}\right)$ one has:

Then

$$
\sum_{\gamma^{\prime} \leqq \gamma}\left|\partial^{\gamma^{\prime}} u\right| \in L^{1} \cap L^{2}\left(\mathbb{R}^{2}\right)
$$

$$
|\alpha(z)|=0\left(\frac{1}{1+|\operatorname{Im} z|^{\gamma_{1}}+|\operatorname{Re} z \operatorname{Im} z|^{\gamma_{2}}}\right) .
$$

Proof. Note that $|\operatorname{Im} z|=\pi\left|\xi_{0}^{1}(z)\right|$ and $|\operatorname{Re} z \operatorname{Im} z|=\pi\left|\xi_{0}^{2}(z)\right|$. Since $|\alpha(z)|=$ $\left|[u m]\left(\xi_{0}(z), z\right)\right|$ this theorem is a consequence of the statement:

For all $\gamma^{\prime} \leqq \gamma,\left|\xi^{\gamma^{\prime}}[u m]^{\wedge}(\xi, z)\right| \in L^{\infty}\left(\mathbb{R}^{2} \times \mathbb{C}\right)$. This will be true exactly when $[u m]^{-}$ decays, which is to say $u m$ is smooth. Since $u$ is smooth to order $\gamma$ by hypothesis, it remains to show that $m$ inherits this smoothness. This is easily established by induction. Suppose $\partial^{\gamma^{\prime}} m \in L^{\infty}$ for all $\gamma^{\prime}<\gamma$. Then

$$
\partial^{\gamma} m=\partial^{\gamma}(I-G)^{-1} 1=\left[\partial^{\gamma},(I-G)^{-1}\right] 1=(I-G)^{-1}\left[\partial^{\gamma}, G\right] m .
$$

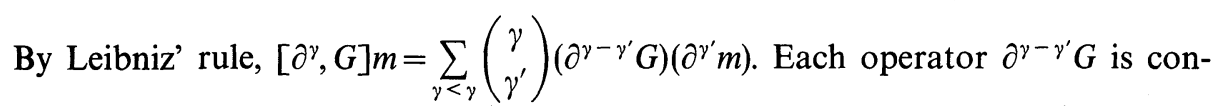
tinuous from $L^{\infty}$ to $L^{\infty}$ because of the smoothness of $u$, while each term $\partial^{\gamma^{\prime}} m$ belongs to $L^{\infty}$ by the induction hypothesis. Finally, $(I-G)^{-1}$ is bounded on $L^{\infty}$ because $u$ is small in $L^{1} \cap L^{2}$. Thus $\partial^{y} m$ is bounded.

Corollary 5.II. Suppose that $\|u\|_{L^{1}}+\|u\|_{L^{2}}$ is small and that $u(x)$ has two continuous derivatives in $L^{1} \cap L^{2}\left(\mathbb{R}^{2}\right)$. Then the scattering data $\alpha(z)$ belongs to the weight space $L^{2}(|\operatorname{Im} z| d \bar{z} \wedge d z)$ and is bounded. 
Proof. Boundedness is a consequence of Corollary 2.III. By Theorem 4.III,

$$
|\alpha(z)| \leqq \frac{c}{1+|\operatorname{Im} z|^{2}+|\operatorname{Re} z \operatorname{Im} z|^{2}} .
$$

With this estimate and a change of variables,

$$
\begin{aligned}
\int_{\mathbb{C}}|\alpha(z)|^{2}|\operatorname{Im} z| d \bar{z} \wedge d z & \leqq c \int_{\mathbb{C}} \frac{|\operatorname{Im} z| d \bar{z} \wedge d z}{\left(1+|\operatorname{Im} z|^{2}+|\operatorname{Re} z \operatorname{Im} z|^{2}\right)^{2}} \\
& =c \int_{\mathbb{R}^{2}} \frac{d x d y}{\left(1+x^{2}+y^{2}\right)^{2}}<\infty . \square
\end{aligned}
$$

As constructed, the forward scattering transform $u \rightarrow T$, or $u \rightarrow \alpha_{0}$ is continuous near 0 as a map from a Sobolev subspace of $L^{1} \cap L^{2}\left(\mathbb{R}^{2}\right)$ to $L^{\infty} \cap L^{2}(|\operatorname{Im} z| d \bar{z} \wedge d z)$. Later it will be shown to be a bijection between subsets of these Banach spaces.

Suppose that $u(x)$ is a small potential in the sense of the preceding corollary. Then the heat operator perturbed by $u$ has the associated scattering operator $T$, and its unique solution $m$ satisfies $\partial_{\bar{z}} m=T m$. The general solution is given by the Fredholm integral equation:

$$
m=\text { "entire" }+C T m \text {. }
$$

Here "entire" denotes a function of $x$ and $z$ which is entire holomorphic in $z$, and $C$ is the Cauchy integral operator, which agrees on the Schwartz class with

$$
C f(z)=\frac{1}{2 \pi i} \int_{\mathbb{C}} \frac{f(\zeta)}{z-\zeta} d \bar{\zeta} \wedge d \zeta
$$

The fact that $m$ is a solution of the heat operator determines "entire," and it can be shown that the equation has a unique solution. This requires some technical results.

Lemma 6.III. Suppose $T f(x, z)=\alpha(z) e^{2 \pi i x \cdot \xi(z)} f(x, \bar{z})$ with $\alpha(z) \in L^{2}(|\operatorname{Im} z| d \bar{z} \wedge d z)$ and bounded. Then $C T$ is continuous from $L^{\infty}\left(\mathbb{R}^{2} \times \mathbb{C}\right)$ to itself and yields functions with some decay in $z$ :

$$
|C T f(x, z)| \leqq C_{z}\left(\|\alpha\|_{L^{\infty}}+\|\alpha\|_{L^{2}(\operatorname{Im} z \mid)}\right)\|f\|_{L^{\infty}},
$$

where $C_{z}$ is a bounded function of $z$ and $\lim _{|\operatorname{lm} z| \rightarrow \infty} C_{z}=0$.

Proof. Taking absolute values inside immediately shows

$$
|C T f(x, z)| \leqq\|f\|_{L^{\infty}}\left[\frac{1}{2 \pi} \int_{C} \frac{|\alpha(\zeta)|}{|z-\zeta|} d \bar{\zeta} \wedge d \zeta\right] .
$$

Changing variables as follows will provide ready-made estimates:

$$
\zeta(\xi)=\frac{\xi_{2}}{2 \xi_{1}}-i \pi \xi_{1},\left|\frac{\partial(\bar{\zeta}, \zeta)}{\partial\left(\xi_{1}, \xi_{2}\right)}\right|=\left|\frac{i \pi}{\xi_{1}}\right| .
$$

A simple calculation shows that $\|\alpha \circ \zeta\|_{L^{\infty}}=\|\alpha\|_{L^{\infty}}$, and that $\|\alpha \circ \zeta\|_{L^{2}\left(\mathbb{R}^{2}\right)}=$ 
$\pi^{-1}\|\alpha\|_{L^{2}(|\operatorname{lm} z|)}$. Meanwhile, the integral to be estimated is transformed into

$$
2 \pi \int_{\mathbb{R}^{2}} \frac{|\alpha \circ \zeta(\xi)|}{\left|P_{z}(\xi)\right|} d \xi
$$

But this is bounded by $C_{z}\left(\left\|\alpha^{\circ} \zeta\right\|_{L^{\infty}}+\left\|\alpha^{\circ} \zeta\right\|_{L^{2}}\right)$ as in Lemma 2.II, hence it is bounded by

$$
C_{z}\left(\|\alpha\|_{L^{\infty}}+\|\alpha\|_{L^{2}(\operatorname{Im} z \mid)}\right) .
$$

Corollary 7.III. If $T$ is given with $\alpha(z)$ small enough in $L^{\infty} \cap L^{2}(|\operatorname{Im} z|)$, then $C T$ is a contraction of $L^{\infty}\left(\mathbb{R}^{2} \times \mathbb{C}\right)$.

Returning to the original problem, suppose $m(x, z)$ is the unique solution to the perturbed heat operator. Then both $m$ and $C T m$ are bounded, so Liouville's theorem guarantees that "entire" in Eq. (III.15) has no $z$-dependence. Hence one can rewrite the equation with some bounded function $q(x)$ replacing "entire":

$$
m(x, z)=q(x)+C T m(x, z) .
$$

Applying the original operator $P(\partial+v)$ to this $m$ yields:

$$
u m=P(\partial+v) m=P(\partial+v) q+P(\partial+v) C T m .
$$

Since $C T m$ is an absolutely convergent integral, it may be differentiated in the parameter $x$. This helps justify two formal calculations, which are tagged for future reference:

\section{Lemma 8.III.}

(i) $[P(\partial+v), T]=0$,

(ii) $[P(\partial+v), C] f(x, z)=\frac{1}{i \pi} \partial_{1} \int_{\mathbb{C}} f(x, z) d \bar{z} \wedge d z$. This is independent of $z$.

Using this lemma, one obtains:

$$
\begin{aligned}
u m & =P(\partial+v) q+C T(P(\partial+v) m)+[P(\partial+v), C T] m \\
& =P(\partial+v) q+u C T m+\frac{1}{\pi i} \partial_{1} \int_{C} T m d \bar{z} \wedge d z .
\end{aligned}
$$

Expanding $P(\partial+v) q$ shows that the only term which grows as $|z| \rightarrow \infty$ is $2 z \partial_{1} q$. Hence $\partial_{1} q=0$. Taking this into account, rewrite the equation as:

$$
m(x, z)-1=q\left(x_{2}\right)-1+\operatorname{CTm}(x, z) .
$$

Fix $x_{2}$, and let $\varepsilon>0$ be given. Let $z$ be chosen with $|\operatorname{Im} z|$ so large that $|\operatorname{CTm}(x, z)|$ $<(\varepsilon / 2)$. For this $z$, choose $x_{1}$ so large that $|m(x, z)-1|<(\varepsilon / 2)$. Thus $\left|q\left(x_{2}\right)-1\right|<\varepsilon$, and since $\varepsilon$ and $x_{2}$ were arbitrary, $q(x) \equiv 1$. The following theorem sums up the preceding argument:

Theorem 9.III. Suppose that $u(x)$ is small in $L^{1} \cap L^{2}\left(\mathbb{R}^{2}\right)$ and that $\partial^{\gamma} u \in L^{1} \cap L^{2}\left(\mathbb{R}^{2}\right)$ for 
all $|\gamma| \leqq 2$. Then the unique solution $m(x, z)$ to the equation

$$
\left[\partial_{1}^{2}+2 z \partial_{1}-\partial_{2}-u(x)\right] m(x, z)=0 \quad \lim _{|x| \rightarrow \infty} m(x, z)=1
$$

is also the unique solution to the equation

$$
m(x, z)=1+C \operatorname{Tm}(x, z)
$$

where $T$ is the scattering data associated to $u$ by the heat operator.

\section{Recovering the Potential from the Scattering Data}

The usefulness of the inverse scattering method hinges on two observations. First, any small scattering operator $T$ of the form in Lemma 6.III determines a unique solution $m(x, z)$ to $m=1+C T m$ which also solves the perturbed heat Eq. (II.3) with some potential $u(x)$. In fact, this inverse transform $T \rightarrow u$ is an analytic Banach space valued map defined on a neighborhood of 0 in a weighted-norm subspace of $L^{\infty} \cap L^{2}(|\operatorname{Im} z| d \bar{z} \wedge d z)$. Second, rather trivial flows of $T$ correspond to interesting nonlinear evolutions of $u$, some of which will be catalogued in the next chapter. The trivial flows have solutions bounded and small for all time, so that by continuity of the inverse map, one obtains global existence theorems for the nonlinear flows of $u$.

Define the "translated" derivatives:

$$
D_{1}=\frac{\partial}{\partial x_{1}}+z, \quad D_{2}=\frac{\partial}{\partial x_{2}}+z^{2}, \quad z \in \mathbb{C} .
$$

Then the heat operator which annihilates $m$ can be written:

$$
\left[D_{1}^{2}-D_{2}-u(x)\right] m(x, z)=0 .
$$

These translated derivatives commute with $T$ in the sense below:

Lemma 1.IV. If $f(x, z)$ has one continuous $x$ derivative, and $T$ has the form

$$
T f(x, z)=\alpha(z) e^{2 \pi i x \cdot \xi_{0}(z)} f(x, \bar{z}),
$$

then $\left[D_{1}, T\right] f=\left[D_{2}, T\right] f=0$.

Proof. A simple calculation.

It is also of value to calculate the commutators of the translated derivatives $D_{1}, D_{2}$ with the Cauchy integral operator $C$. Since both $\partial / \partial x_{1}$ and $\partial / \partial x_{2}$ commute with $C$, it suffices to calculate $\left[z^{n}, C\right]$. More precisely:

Lemma 2.IV. Suppose $f(x, z)$ is a Schwartz function over $\mathbb{R}^{2} \times \mathbb{C}$, and let $C$ denote the Cauchy integral operator in the z-variable. Then

$$
\begin{gathered}
{\left[z^{n}, C\right] f(x, z)=\frac{1}{2 \pi i} \sum_{k=0}^{n-1} z^{k} \int_{\mathbb{C}} \zeta^{n-k-1} f(x, \zeta) d \bar{\zeta} \wedge d \zeta,} \\
{\left[D_{1}^{n}, C\right] f(x, z)=\sum_{k=0}^{n}\left(\begin{array}{l}
n \\
k
\end{array}\right) \partial_{1}^{n-k}\left[z^{k}, C\right] f(x, z) .}
\end{gathered}
$$


Proof. Part (IV.5) is the binomial theorem. Part (IV.4) follows from the identity

$$
z^{n}-\zeta^{n}=(z-\zeta) \sum_{k=0}^{n-1} z^{k} \zeta^{n-k-1}
$$

This reduces $\left[z^{n}, C\right] f=(1 / 2 \pi i) \int_{\mathbb{C}}\left(z^{n}-\zeta^{n}\right)(z-\zeta)^{-1} f d \bar{\zeta} \wedge d \zeta$ to the desired form.

Schwartz-class regularity is not necessary for either of the preceding lemmas - it is invoked to smooth out the exposition. The formulas to be derived will have obvious regularity requirements, which will be much weaker. Note that these lemmas imply Lemma 8.III.

Returning to the original discussion, suppose that $T$ is an operator of the form in Lemma 1.IV. The following might be called the inverse scattering theorem.

Theorem 3.IV. If $T$ is given by a function $\alpha(z)$ that is small in $L^{\infty} \cap L^{2}(|\operatorname{Im} z| d \bar{z} \wedge d z)$, then the unique solution to $m=1+C T m$ solves the perturbed heat equation $P(\partial+v) m$ $=u m$ for a potential $u(x)$ which may be represented by the formula:

$$
u(x)=\frac{1}{\pi i} \partial_{1} \int_{\mathbb{C}} T m(x, z) d \bar{z} \wedge d z .
$$

Proof. Corollary 7.III and the contraction mapping theorem guarantee a unique solution to $m=1+C T m$. Applying $P(\partial+v)$ to both sides as in Eq. (III.21) and using Lemma 2.IV yields:

$$
P(\partial+v) m=C T P(\partial+v) m+\frac{1}{\pi i} \partial_{1} \int_{\mathbb{R}} T m(x, z) d \bar{z} \wedge d z .
$$

This last term-call it $u(x)$ - has no $z$-dependence. Thus

$$
P(\partial+v) m=u(x)(I-C T)^{-1} 1=u(x) m .
$$

Of course, the integral representing $u$ need not make sense without additional hypotheses on $\alpha(z)$. There is no problem if $\alpha$ is of the Schwartz class, but something much less drastic works, too. To develop these hypotheses, it is convenient to construct instead the Fourier transform (in $x$ ) of the function $m(x, z)-1$ :

$$
[m-1] \hat{\jmath}=[C T(m-1) \hat{\jmath}+[C T 1 \hat{\jmath} .
$$

It is time to point out that the function $z \rightarrow \xi_{0}(z)$ and $\xi \rightarrow \zeta(\xi)$ defined in Eqs. (II.7) and (III.18) are inverses of each other. Thus

$$
\begin{aligned}
& {[C T 1] \hat{\top}(\xi, z)=\left[\frac{1}{2 \pi i} \int_{\mathbb{C}}^{\alpha(\zeta) e^{2 \pi i x \cdot \xi_{0}(\zeta)}} \frac{z \zeta}{z-\zeta} \wedge d \zeta\right](\xi, z)} \\
& =\left[2 \pi i \int_{\mathbb{R}^{2}} \frac{\alpha \circ \zeta(\xi) e^{2 \pi i x \cdot \xi}}{P_{z}(\xi)} d \xi\right](\xi, z)=\frac{2 \pi i \alpha \circ \zeta(\xi)}{P_{z}(\xi)} .
\end{aligned}
$$


Likewise, if $f$ is in the Schwartz class,

$$
\begin{aligned}
{[C T f]^{\hat{y}}(\xi, z) } & =\left[\frac{1}{2 \pi i} \int_{\mathbb{C}} \frac{\alpha(\zeta) f(x, \zeta) e^{2 \pi i x \cdot \xi_{0}(\xi)}}{z-\zeta} d \bar{\zeta} \wedge d \zeta\right] \\
& =\frac{1}{2 \pi i} \int_{\overparen{C}} \frac{\alpha(\zeta)}{z-\zeta} \hat{f}\left(\xi-\xi_{0}(\zeta), \bar{\zeta}\right) d \bar{\zeta} \wedge d \zeta \equiv R_{\alpha} \hat{f}(\xi, z) .
\end{aligned}
$$

If the $L^{1}\left(\mathbb{R}^{2}\right)$ norm of $\hat{f}(\xi, z)$ is a bounded function of $z$, then so is the $L^{1}\left(\mathbb{R}^{2}\right)$ norm of $R_{\alpha} \hat{f}(\xi, z)$. This may be seen by integrating Eq. (IV.11) first in $\xi$. Under the hypothesis that $\alpha$ is small in $L^{\infty} \cap L^{2}(|\operatorname{Im} z|)$, the map $\hat{f} \rightarrow R_{\alpha} \hat{f}$ is a contraction of $L^{1}\left(\mathbb{R}^{2}\right)$ for every $z \in \mathbb{C}$. Likewise, $\left(2 \pi i \alpha \circ \zeta(\xi) / P_{z}(\xi)\right)$ is in $L^{1}\left(\mathbb{R}^{2}\right)$ uniformly in $z$. Thus Eq. (IV.9) has a unique solution $[m-1]^{\hat{(}}(\xi, z)$ in $L^{1}\left(\mathbb{R}^{2}\right)$ uniformly in $z$. But its inverse Fourier transform $m(x, z)$ must be the unique solution to $m=1+C T m$. This fact strengthens Theorem 3.IV with the expected result that for each $z \in \mathbb{C}$, $\lim m(x, z)=1$. $|x| \rightarrow \infty$

Using Eqs. (IV.10) and (IV.11), it is possible to write down the Fredholm integral equation for $[m-1]$ more explicitly:

$$
[m-1] \hat{(\xi, z)}=\frac{2 \pi i \alpha(\zeta(\xi))}{P_{z}(\xi)}+R_{\alpha}([m-1])(\xi, z) .
$$

Defining:

$$
\|\alpha\|_{L^{\infty} \cap L^{2}(\operatorname{Im} z \mid)}=\max \left(C_{z}\right)\left[\|\alpha\|_{L^{\infty}}+\|\alpha \circ \zeta\|_{L^{2}}\right],
$$

where $C_{z}$ is the coefficient in Lemma 2.II, and recalling the integrability of $\left(\alpha \circ \zeta / P_{z}\right)$ and the boundedness of $R_{\alpha}$ on $L^{1}\left(\mathbb{R}^{2}\right)$, it is possible to give an explicit estimate:

$$
\|[m-1] \hat{(\cdot, z)}\|_{L^{1}\left(\mathbb{R}^{2}\right)} \leqq \frac{\|\alpha\|_{L^{\infty} \cap L^{2}(\operatorname{Im} z \mid)}}{1-\|\alpha\|_{\left.L^{\infty} \cap L^{2}|\operatorname{Im} z|\right)}}
$$

Observe that this is independent of $z$. Thus, if $K_{-[m-1]^{-}}$denotes the operator "convolution in $\xi$ by $-[m-1](\xi, z)$," it can be estimated uniformly in $z$ :

$$
\left\|K_{-[m-1]^{-}}\right\|_{L^{p}\left(\mathbb{R}^{2}\right) \rightarrow L^{p}\left(\mathbb{R}^{2}\right)} \leqq \frac{\|\alpha\|_{L^{\infty} \cap L^{2}(\operatorname{Im} z \mid)}}{1-\|\alpha\|_{L^{\infty} \cap L^{2}(I \mathrm{Im} z \mid)}} \text { for } \quad 1 \leqq p \leqq \infty .
$$

This estimate will be used to bound $\hat{u}(\xi)$ in $L^{2}\left(\mathbb{R}^{2}\right)$ and $L^{\infty}\left(\mathbb{R}^{2}\right)$. From Theorem 3.IV, one obtains a Fredholm integral equation for $\hat{u}$ :

$$
\begin{aligned}
& P_{z}(\xi)[m-1] \hat{(\xi, z)}=[u m]^{\hat{u}}=\hat{u} *[m-1] \hat{(\xi, z)+\hat{u}(\xi)} \\
& \Rightarrow \hat{u}(\xi)=K_{-[m-1]^{(}}(\hat{u})(\xi, z)+P_{z}(\xi)[m-1]^{(\xi, z) .}
\end{aligned}
$$

It is a remarkable feature of scattering theory that the $z$-dependence of the righthand side of (IV.16) cancels itself out. However, Eq. (IV.15) and results below will yield estimates on $\hat{u}$ that are independent of $z$.

It remains to estimate $P_{z}[m-1]^{\wedge}$ in $L^{2}$ and $L^{\infty}$. This may be done from Eq. (IV.12) multiplied by $P_{z}$ :

$$
P_{z}[m-1] \hat{\jmath}=2 \pi i \alpha \circ \zeta+P_{z} R_{\alpha}[m-1] \hat{~}
$$


This is yet another Fredholm integral equation because of the following technical lemma:

Lemma 4.IV. Suppose $f(\xi, z)$ is in the Schwartz class over $\mathbb{R}^{2} \times \mathbb{C}$. Then two identities hold:

$$
\begin{aligned}
R_{\alpha} f & =R_{\left(\alpha / P_{z}\right)}\left(P_{z} f\right), \\
P_{z} R_{\left(\alpha / P_{z}\right)} f & =\left(R_{\alpha} f\right)(\xi, z)-\left(R_{\alpha} f\right)(\xi, \zeta(\xi)) .
\end{aligned}
$$

Proof. For Eq. (IV.18), observe:

$$
R_{\left(\alpha / P_{z}\right)}\left(P_{z} f\right)=\frac{1}{2 \pi i} \int_{\mathbb{C}} \frac{\alpha(\zeta)}{P_{\zeta}(\xi)} P \bar{\zeta}\left(\xi-\xi_{0}(\zeta)\right) f\left(\xi-\xi_{0}(\zeta), \bar{\zeta}\right) \frac{d \bar{\zeta} \wedge d \zeta}{z-\zeta}
$$

The result follows from identity (II.15): simply cancel the two polynomials. Likewise, Eq. (IV.19) follows from the identity:

$$
\frac{P_{z}(\xi)}{(z-\zeta) P_{\zeta}(\xi)}=\frac{1}{z-\zeta}-\frac{1}{\zeta(\xi)-\zeta} . \square
$$

Thus, defining the operator $M_{\alpha}$ by the right-hand side of Eq. (IV.19), one can write:

$$
P_{z}[m-1]^{\hat{\prime}}=2 \pi i \alpha \circ \zeta+M_{\alpha}\left(P_{z}[m-1]^{\wedge}\right) .
$$

Unfortunately, $M_{\alpha}$ is not continuous on $L^{2} \cap L^{\infty}\left(\mathbb{R}^{2}\right)$ without further restrictions on $\alpha(z)$. These restrictions may be stated as membership in an appropriate subspace of $L^{\infty} \cap L^{2}(|\operatorname{Im} z| d \bar{z} \wedge d z)$, as defined below:

Definition 5.IV. Let $k$ be a nonnegative integer, and write

$$
\langle\xi\rangle^{k}=(1+|\xi|)^{k} \text {. }
$$

Then the $k^{\text {th }}$ weighted subspace of $L^{\infty} \cap L^{2}\left(\mathbb{R}^{2}\right)$ may be denoted by

$$
W^{k}=W^{k}\left(L^{\infty} \cap L^{2}\left(\mathbb{R}^{2}\right)\right)=\left\{f(\xi) \in L^{\infty} \cap L^{2}\left(\mathbb{R}^{2}\right):\langle\xi\rangle^{k} f(\xi) \in L^{\infty} \cap L^{2}\left(\mathbb{R}^{2}\right)\right\} .
$$

This is a Banach space with the norm

$$
\|f\|_{W^{k}}=\sum_{j=0}^{k}\left(\begin{array}{c}
k \\
j
\end{array}\right)\left\|\langle\xi\rangle^{j} f(\xi)\right\|_{L^{\infty} \cap L^{2}\left(\mathbb{R}^{2}\right)}
$$

Here

$$
\|f\|_{L^{\infty} \cap L^{2}\left(\mathbb{R}^{2}\right)}=\|f\|_{L^{\infty}}+\|f\|_{L^{2}} .
$$

Definition 6.IV. The $k^{\text {th }}$ weighted subspace of $L^{\infty} \cap L^{2}(|\operatorname{Im} z| d \bar{z} \wedge d z)$, denoted $W_{\zeta}^{k}=W_{\zeta}^{k}\left(L^{\infty} \cap L^{2}\right)(|\operatorname{Im} z| d \bar{z} \wedge d z)$, consists of those functions $\alpha(z)$ for which $\alpha \circ \zeta(\xi) \in W^{k}\left(L^{\infty} \cap L^{2}\left(\mathbb{R}^{2}\right)\right)$. This is a Banach space with the norm $\|\alpha\|_{W_{\zeta}^{k}}=$ $\|\alpha \circ \zeta\|_{W^{k}}$.

Definition 7.IV. If $f(\xi, z) \in L^{\infty}\left(\mathbb{R}^{2} \times \mathbb{C}\right)$, introduce the maximal function

$$
f^{*}(\xi)=\underset{z \in \mathbb{C}}{\operatorname{ess} \sup }|f(\xi, z)| .
$$


Then define the $k^{\text {th }}$ weighted mixed norm subspace

$$
\begin{aligned}
W_{\infty}^{k}=W_{\infty}^{k}\left(L^{\infty}\left(\mathbb{R}^{2} \times \mathbb{C}\right)\right)= & \left\{f \in L^{\infty}\left(\mathbb{R}^{2} \times \mathbb{C}\right):\langle\xi\rangle^{j} f^{*}(\xi)\right. \text { is } \\
& \text { essentially bounded for all } 0 \leqq j \leqq k\} .
\end{aligned}
$$

This is a Banach space with the norm

$$
\|f\|_{W_{\infty}^{k}}=\max _{0 \leqq j \leqq k}\left\|\langle\xi\rangle^{j} f^{*}(\xi)\right\|_{L^{\infty}\left(\mathbb{R}^{2}\right)}
$$

Abusing notation, define $(f(\xi))^{*} \equiv|f(\xi)|$. Then it is clear that $W^{k} \subset W_{\infty}^{k}$, with $\|f\|_{W_{\infty}^{k}} \leqq\|f\|_{W^{k}}$. Likewise, since $\left(1 /\langle\xi\rangle^{2}\right)$ belongs to $L^{2}\left(\mathbb{R}^{2}\right)$ with norm less than 2, one can say that $W_{\infty}^{k+2} \subset W^{k}$ in the sense that if $f \in W_{\infty}^{k+2}$, then $f^{*} \in W^{k}$. A crude comparison of norms shows:

$$
\left\|f^{*}\right\|_{W^{k}} \leqq \sum_{j=0}^{k}\left(\begin{array}{c}
k \\
j
\end{array}\right)\left\|\frac{f \|_{W_{\infty}^{k+2}}}{\langle\xi\rangle^{2}}\right\|_{L^{\infty} \cap L^{2}\left(\mathbb{R}^{2}\right)} \leqq 3 \cdot 2^{k}\|f\|_{W_{\infty}^{k+2}}
$$

Returning to the original problem, one obtains a bound for $M_{\alpha}$ on these subspaces:

Lemma 8.IV. If $\alpha \in W_{\zeta}^{k}$ and $f \in W_{\infty}^{k}$, then

$$
M_{\alpha} f \in W_{\infty}^{k}
$$

and

$$
\left\|M_{\alpha}\right\|_{W_{\infty}^{k} \rightarrow W_{\infty}^{k}} \leqq 4 \pi\|\alpha\|_{W_{\zeta}^{k}}
$$

Proof. Equation IV.19 and the triangle inequality give

$$
\left\|M_{\alpha}\right\|_{W_{\infty}^{k}} \leqq 2\left\|R_{\alpha} f\right\|_{W_{\infty}^{k}} .
$$

The binomial theorem yields the inequality:

$$
\langle\xi\rangle^{k} \leqq \sum_{j=0}^{k}\left(\begin{array}{l}
k \\
j
\end{array}\right)\left\langle\xi-\xi^{\prime}\right\rangle^{j}\left\langle\xi^{\prime}\right\rangle^{k-j}
$$

Thus

$$
\begin{aligned}
& \left|\langle\xi\rangle^{k} R_{\alpha} f(\xi, z)\right| \leqq \frac{1}{2 \pi} \int\left|\frac{\alpha(\zeta)\langle\xi\rangle^{k}}{z-\zeta} f\left(\xi-\xi_{0}(\zeta), \bar{\zeta}\right)\right| d \bar{\zeta} \wedge d \zeta \\
& \leqq \frac{1}{2 \pi} \sum_{j=0}^{k}\left(\begin{array}{c}
k \\
j
\end{array}\right) \int_{\mathbb{C}}\left|\frac{\alpha(\zeta)\left\langle\xi_{0}(\zeta)\right\rangle^{j}}{z-\zeta}\right|\left|\left\langle\xi-\xi_{0}(\zeta)\right\rangle^{k-j} f\left(\xi-\xi_{0}(\zeta), \bar{\zeta}\right)\right| d \bar{\zeta} \wedge d \xi \\
& \leqq 2 \pi\|f\|_{W_{\infty}^{k}} \sum_{j=0}^{k}\left(\begin{array}{l}
k \\
j
\end{array}\right) \iint_{\mathbb{R}^{2}}\left|\frac{\alpha \circ \zeta(\xi)\langle\xi\rangle^{j}}{P_{z}(\xi)}\right| d \xi \\
& \leqq 2 \pi\|f\|_{W_{\infty}^{k}} \sum_{j=0}^{k}\left(\begin{array}{l}
k \\
j
\end{array}\right)\left\|\langle\xi\rangle^{j} \alpha \circ \zeta(\xi)\right\|_{L^{\infty} \cap L^{2}\left(\mathbb{R}^{2}\right)} \\
& =2 \pi\|f\|_{W_{\infty}^{k}}\|\alpha\|_{W_{\zeta}^{k}} \text {. }
\end{aligned}
$$

Using Eq. (IV.20) and the contraction mapping theorem in $W_{\infty}^{k}$, one obtains the 
estimate:

$$
\| P_{z}[m-1] \hat{\|_{W_{\infty}^{k}}} \leqq \frac{2 \pi\|\alpha \circ \zeta\|_{W_{\infty}^{k}}}{1-4 \pi\|\alpha\|_{W_{\zeta}^{k}}}
$$

Comparing norms with Eq. (IV.22) and observing that $\|f\|_{L^{\infty} \cap L^{2}\left(\mathbb{R}^{2}\right)}=\|f\|_{W^{0}}$, one obtains (for $k=2$ ):

$$
\left\|P_{z}[m-1] \hat{\wedge}\right\|_{L^{\infty} \cap L^{2}\left(\mathbb{R}^{2}\right)} \leqq \frac{72 \pi\|\alpha\|_{W_{\zeta}^{2}}}{1-4 \pi\|\alpha\|_{W_{\zeta}^{2}}}
$$

This estimate is uniform in $z$. Putting it together with Eqs. (IV.15) and (IV.16) yields

$$
\|\hat{u}\|_{L^{\infty} \cap L^{2}} \leqq \frac{\left\|P_{z}[m-1]^{2}\right\|_{L^{2} \cap L^{\infty}\left(\mathbb{R}^{2}\right)}}{1-\left\|K_{-[m-1]^{-}}\right\|_{L^{2} \cap L^{\infty}\left(\mathbb{R}^{2}\right)}} \leqq\left(\frac{1-\|\alpha\|_{L^{2} \cap L^{\infty}(\operatorname{Im} z \mid)}}{1-2\|\alpha\|_{L^{2} \cap L^{\infty}(\operatorname{Im} z)}}\right)\left(\frac{72 \pi\|\alpha\|_{W_{\zeta}^{2}}}{1-4 \pi\|\alpha\|_{W_{\zeta}^{2}}}\right) .
$$

This result may be summed up as follows:

Theorem 9.IV. Suppose that $\alpha(z)$ is bounded and that $\left[1+(\operatorname{Im} z)^{2}+(\operatorname{Re} z \operatorname{Im} z)^{2}\right] \alpha(z)$ is small in $L^{\infty} \cap L^{2}(|\operatorname{Im} z| d \bar{z} \wedge d z)$. Then there is a function $u(x) \in L^{2}\left(\mathbb{R}^{2}\right)$ with bounded Fourier transform, such that $\alpha(z)$ is the scattering data associated to $u$.

Proof. The decay condition implies that $\alpha \in W_{\zeta}^{2}$, as well as that $\alpha \in L^{\infty} \cap L^{2}(|\operatorname{Im} z| d \bar{z} \wedge d z)$. Form the scattering operator $T$ and let $m(x, z)$ be the unique solution to $m=1+C T m$ guaranteed by Theorem 3.IV. This $m$ is a solution to Eq. (IV.2), $P(\partial+v) m=u m$, with $u(x)$ given by $(1 / i \pi) \partial_{1} \int_{\mathbb{C}} T m(x, z) d \bar{z} \wedge d z$. By Eq. (IV.25) and Plancherel's theorem, $u$ is small in $L^{2}\left(\mathbb{R}^{2}\right)$ and has small bounded Fourier transform. By the proof of Theorem 1.II, this guarantees that there is exactly one solution $m_{0}$ to Eq. (IV.2) that has the property that $\left[m_{0}-1\right\}$ is integrable. This fact allows the extension of the forward scattering transform to the ball $\left\{u \in L^{2}\left(\mathbb{R}^{2}\right)\right.$ : $\left.\|u\|_{L^{2}}+\|\hat{u}\|_{L^{\infty}}<\varepsilon\right\}$ by using $T_{0} m_{0}=(\partial / \partial \bar{z}) m_{0}$. This extension is well defined and agrees with Definition 3.III on the ball $\left\{u \in L^{2}\left(\mathbb{R}^{2}\right):\|u\|_{L^{2}}+\|u\|_{L^{1}}<1\right\}$.

Now $m$ solves Eq. (IV.2), and by Eq. (IV.14) it also has the property that $[m-1] \in L^{1}\left(\mathbb{R}^{2}\right)$. By uniqueness, $m=m_{0}$, and therefore the (extended) scattering operator $T_{0}$ associated to $u$ is the same as $T$.

\section{Evolutions of the Heat Operator}

The preceding discussion leads to a method of solving the initial value problem for a large class of nonlinear evolution equations. For certain functions $K$, described below, one seeks a solution $u=u(x, t)$ to the problem

$$
\frac{d}{d t} u=K(u), \quad u(x, 0)=u_{0}(x)
$$

If $t \rightarrow u(x, t)$ is continuous on $t \geqq 0$, then $u(x, t)$ is called a global solution. Otherwise, one might hope that for some $\tau>0$, the map $t \rightarrow u(x, t)$ is continuous for $0 \leqq t \leqq \tau$. This is a local solution. 
Considering $u$ to be a potential for the heat operator reduces the question of existence of a solution to the perhaps simpler existence problem for another evolution equation:

$$
\begin{aligned}
& u(x, 0) \stackrel{\substack{\text { forward } \\
\text { scattering }}}{\longrightarrow} \alpha(z, 0) \\
& u(x, t) \stackrel{\substack{\text { inverse } \\
\text { scattering }}}{\longleftarrow} \alpha(z, t)
\end{aligned}
$$

In fact, for certain functions $K$, if $u$ evolves like Eq. (V.1), then $\alpha=\alpha(z, t)$ satisfies a linear evolution equation.

Continuity for the forward and inverse scattering maps means the continuity of all the maps represented by arrows connecting two descending chains of Banach spaces:

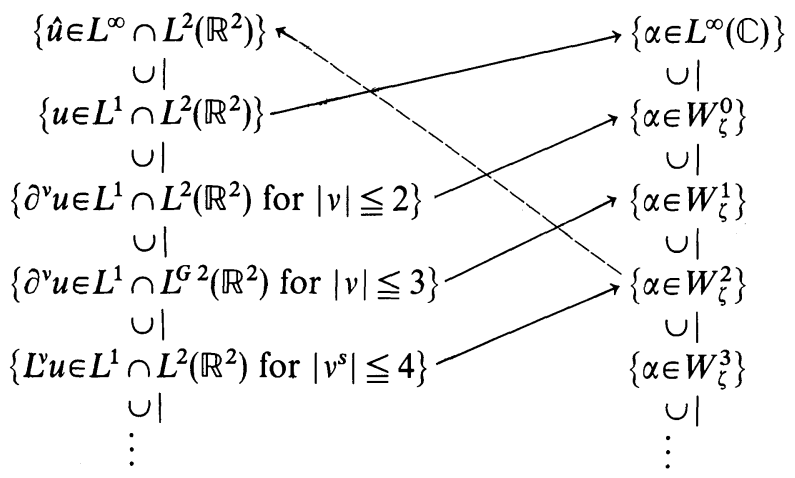

Solid arrows represent the forward scattering maps - their "slope" is 1 by Theorem 4.III. The dashed arrow represents the inverse scattering map-its slope is determined by Theorem 9.IV. The weighted-norm spaces $W_{\zeta}^{k}$ are described by Definition 6.IV.

These Banach spaces are natural for the potentials and scattering data related by the perturbed heat operator. Theirs are the topologies which underlie the notion of a continuous evolution $t \rightarrow u(\cdot, t)$ or $t \rightarrow \alpha(\cdot, t)$.

One class of nonlinear evolution equations that have global solutions by this method is the Kadomtsev-Petviashvili hierarchy. As defined by Date, Jimbo, Kashiwara and Miwa [DJKM], this is the set of compatibility conditions between the perturbed heat operator $L=\partial_{1}-\partial_{2}-u(x)$ and a list of evolution operators $B_{n} ; n \geqq 3$.

$$
B_{n} \psi=\partial_{1}^{n} \psi+\frac{n}{2} u(x) \partial_{1}^{n-1} \psi+v_{3}(x) \partial_{1}^{n-3} \psi+\cdots+v_{n-1}(x) \partial_{1} \psi+v_{n}(x) \psi .
$$

In other words, the $n^{\text {th }}$ evolution equation in the KP hierarchy is the Lax equation:

$$
\frac{d}{d t} L=\left[B_{n}, L\right]
$$

Since $(d / d t) L=-(d / d t) u$, the left-hand side of Eq. V.5 is a differential operator of 
degree 0 . The right-hand side has degree $n-2$ because of the special form of $B_{n}$. Thus one obtains $n-1$ equations for the $n$ unknown functions $(d / d t) u(x), u(x)$, $v_{3}(x), \ldots, v_{n}(x)$. In principle, one can solve this system by writing $v_{3}, \ldots, v_{n}$ in terms of $u$ and its derivatives, ultimately obtaining $(d / d t) u=K_{n}(u)$ for some function $K_{n}$ of $u$, its derivatives and its antiderivatives. This inconvenient calculation is much facilitated by the no-worse-than bilinear right-hand side of (V.5).

But all such evolutions $K_{n}$ correspond to trivial evolutions of the scattering data. To see, this consider an asymptotically exponential solution $\psi$ to $L \psi=0$, with $u$ small. Suppose that $\psi$ evolves so as to satisfy $(d / d t) \psi=B_{n} \psi$. Then one can write $\psi=m(x, t, z) e^{x_{1} z+x_{2} z^{2}+t z^{n}}$, where $m(x, t, z)$ is bounded in all variables. Thus $m$ satisfies the system below:

$$
\begin{aligned}
& {\left[\left(\partial_{1}+z\right)^{2}-\left(\partial_{2}+z^{2}\right)-u(x)\right] m=0,} \\
& \frac{d}{d t} m=-z^{n} m+\left(\partial_{1}+z\right)^{n} m+\frac{n}{2} u(x)\left(\partial_{1}+z\right)^{n-2} m+\cdots+v_{n}(x) m .
\end{aligned}
$$

In this way the evolution of $u$ results in an evolution of $m$, hence of the asymptotic behavior of $m$.

Lemma V.1. Suppose $m \sim 1+\beta(z) /\left(x_{1}+2 z x_{2}\right)+\alpha(z) e^{2 \pi i x \cdot \xi(z)} /\left(x_{1}+2 \bar{z} x_{2}\right)$ as $|x|$ $\rightarrow \infty$, and that $m$ evolves in time according to the equation

$$
\dot{m}=Q(\partial+v) m-Q(v) m+\sum_{|v|<\operatorname{deg} Q} c_{v}(x)(\partial+v)^{v} m,
$$

where $Q$ is a constant-coefficient polynomial and the $c_{v}$ are test functions. Then

$$
\dot{\alpha}=[Q(\bar{v})-Q(v)] \alpha, \quad \dot{\beta}=0 .
$$

"Proof". On the one hand,

$$
\dot{m}=\frac{\dot{\beta}(z)}{x_{1}+2 z x_{2}}+\frac{\dot{\alpha}(z) e^{2 \pi i x \cdot \xi(z)}}{x_{1}+2 \bar{z} x_{2}}+o\left(\frac{1}{|x|}\right) .
$$

On the other hand, since each $c_{v}$ is a test function, only the constant coefficient terms appear in the asymptotic behavior of $m$ :

$$
\dot{m}=[Q(\partial+v)-Q(v)] m+o\left(\frac{1}{|x|}\right) \text { as }|x| \rightarrow \infty .
$$

Now

$$
\begin{aligned}
Q(\partial+v)\left[\frac{\beta(z)}{x_{1}+2 z x_{2}}\right] & =\frac{Q(v) \beta(z)}{x_{1}+2 z x_{2}}+o\left(\frac{1}{|x|}\right), \\
Q(\partial+v)\left[\frac{\alpha(z) e^{2 \pi i x \cdot \xi(z)}}{x_{1}+2 \bar{z} x_{2}}\right] & =\frac{Q(\bar{v}) \alpha(z) e^{2 \pi i x \cdot \xi(z)}}{x_{1}+2 \bar{z} x_{2}}+o\left(\frac{1}{|x|}\right) .
\end{aligned}
$$

One makes the usual claim that if the remainder is $o(1 /|x|)$, then so are its derivatives. Thus $[Q(\partial+v)-Q(v)] o(1 /|x|)=o(1 /|x|)$. Comparing the leading 
oscillatory and non-oscillatory terms of (V.8) and (V.9) as $|x| \rightarrow \infty$ completes the proof.

In particular, the case $Q(\partial+v)=\left(\partial_{1}+z\right)^{3}$ corresponds to the KP equation, and $Q(\partial+v)=\left(\partial_{1}+z\right)^{n}$ to the rest of the hierarchy.

One might observe that $Q(v)$ need not be a polynomial. Thus it is useful to construct a functional calculus for the scattering transform which will relate evolutions of $\alpha$ to evolutions of $u$. The first step is to construct the tangent map which sends infinitesimal generators of flows of $\alpha$ to infinitesimal generators of flows of $u$. But since

$$
u(x)=\frac{1}{\pi i} \partial_{1} \int_{\mathbb{C}} T m(x, z) d \bar{z} \wedge d z
$$

one has

$$
\begin{aligned}
\pi i \dot{u} & =\partial_{1} \int_{\mathbb{C}}(T m)^{\cdot}=\partial_{1} \int_{\mathbb{C}}\left[T(I-C T)^{-1} 1\right]^{\cdot} \\
& =\partial_{1} \int_{\mathbb{C}}(I-T C)^{-1} \dot{T} m=\partial_{1} \int_{\mathbb{C}}\left[\left(I-C^{t} T^{t}\right)^{-1}\right] \dot{T} m=\partial_{1} \int_{\mathbb{C}} \tilde{m} \dot{T} m, \\
\tilde{m} & =\left(I-C^{t} T^{t}\right)^{-1} 1=(I-C \tilde{T})^{-1} 1
\end{aligned}
$$

with $\widetilde{T}=-T^{t}$, since $C^{t}=-C$. Here, the superscript $t$ denotes transposition with respect to the inner product on $\left(\mathbb{R}^{2} \times \mathbb{C}\right)$ defined by

$$
\langle\phi, \psi\rangle=\int_{\mathbb{R}^{2} \times \mathbb{C}} \phi(x, z) \psi(x, z) d x_{1} d x_{2} d \bar{z} \wedge d z
$$

With respect to this inner product, $\partial^{t}=-\partial$. The function $\tilde{m}(x, z)$ satisfies the transposed equation $P(-\partial+v) \tilde{m}=u \tilde{m}$, as shown below.

First note that $[P(-\partial+v), \widetilde{T}]=[P(\partial+v), T]^{t}=0$, and thus that $P(-\partial+v) \tilde{m}=P(-\partial+v)(I-C \tilde{T})^{-1} 1=(I-C \tilde{T})^{-1}[P(-\partial+v), C] \tilde{T} \tilde{m}=\tilde{u} \tilde{m}$, where $\tilde{u}=\tilde{u}(x)=[P(-\partial+v), C] \tilde{T} \tilde{m}=(1 / \pi i) \partial_{1} \int_{\mathbb{C}}-\tilde{T} \tilde{m} d z \wedge d z$. But $\tilde{u}(x)=u(x)$, since $\int_{\mathbb{C}}-\tilde{T} \tilde{m}=\int_{\mathbb{C}}(I-T C)^{-1} T 1=\int_{\mathbb{C}} T m$. One can transpose $T$ with respect to "half" the inner product because its action on the $x$-variable is so trivial.

This yields the tangent map:

\section{Lemma V.2.}

$$
\dot{u}(x, t)=\frac{1}{\pi i} \partial_{1} \int_{\mathbb{C}} \tilde{m}(x, z) \dot{T} m(x, z) d \bar{z} \wedge d z,
$$

where

$$
P(\partial+v) m=u m, \quad P(-\partial+v) \tilde{m}=u \tilde{m}
$$

or alternatively,

$$
\partial_{\bar{z}} m=T m, \quad \partial_{\bar{z}} \tilde{m}=\tilde{T} \tilde{m} .
$$


Pick a function $\phi(z)$ and consider the initial value problem

$$
\dot{\alpha}(z, t)=[\phi(z)-\phi(\bar{z})] \alpha(z, t), \quad \alpha(z, 0)=\alpha_{0}(z) .
$$

This corresponds to $\dot{T}=\left[M_{\phi}, T\right]$, where $M_{\phi}$ designates the operation of multiplication by $\phi$.

The corresponding evolution of $u$ is given by

$$
\begin{aligned}
& \dot{u}=\frac{1}{\pi i} \partial_{1} \int_{\mathbb{C}} \tilde{m}\left[M_{\phi}, T\right] m d \bar{z} \wedge d z=\frac{1}{\pi i} \partial_{1} \int_{\mathbb{C}} \phi[\tilde{m} T m+(\tilde{T} \tilde{m}) m] d \bar{z} \wedge d z, \\
& \dot{u}=\frac{1}{\pi i} \partial_{1} \int_{\mathbb{C}} \phi \partial_{\bar{z}}(\tilde{m} m) d \bar{z} \wedge d z .
\end{aligned}
$$

Now if for all $z \in \mathbb{C}, \operatorname{Re}[\phi(z)-\phi(\bar{z})] \leqq 1$, then the solution to (V.12) remains bounded in each $W_{\zeta}^{k}$ for all $t$. If in addition $\operatorname{Re}[\phi(z)-\phi(\bar{z})]=0$, then $\|\alpha(z, t)\|_{W_{\zeta}^{k}}=\left\|\alpha_{0}\right\|_{W_{\zeta}^{k}}$ for all $t$. These bounded evolutions of $\alpha$ correspond to bounded evolutions of $u$, and include all the evolutions in the KP hierarchy, as will be shown below:

Let $\phi(z)=(s-z)^{-1}$ for $s \in \mathbb{R}$. Then $\operatorname{Re}[\phi(z)-\phi(\bar{z})]=0$ and $\dot{T}=\left[M_{\phi}, T\right]$ is a bounded evolution. The corresponding evolution of $u(x)$ is

$$
\begin{aligned}
\dot{u} & =\frac{1}{\pi i} \partial_{1} \int_{\mathbb{C}} \phi \partial_{\bar{z}}(\tilde{m} m)=\frac{-1}{\pi i} \partial_{1} \int_{\mathbb{C}}\left(\partial_{z}(s-z)^{-1}\right) \tilde{m} m \\
& =\frac{1}{\pi i} \partial_{1} \int_{\mathbb{C}} \pi i \delta_{s}(z) \tilde{m}(x, z) m(x, z) d \bar{z} \wedge d z=\partial_{1}(\tilde{m}(x, s) m(x, s)) .
\end{aligned}
$$

Hence $u(x)$ solves the nonlinear system

$$
\dot{u}=\partial_{1}(\bar{m} m), \quad\left(\partial_{1}^{2}+2 s \partial_{1}-\partial_{2}\right) m=u m, \quad\left(\partial_{1}^{2}-2 s \partial_{1}+\partial_{2}\right) \tilde{m}=u \tilde{m} .
$$

But since $(s-z)^{-1}=\sum_{n=1}^{\infty} z^{n-1} / s^{n}$ (whenever $\left.|s|>|z|\right)$ this system is equivalent to the KP hierarchy as follows.

Consider the algebra of formal power series in $s^{-1}$ with coefficients in a ring $R$. Let $\lambda_{k}$ be the linear functional on this algebra defined by

$$
\lambda_{k}\left(\sum_{n=0}^{\infty} \frac{r_{n}}{s^{n}}\right)=r_{k+3}, \quad r_{n} \in R
$$

Then $\lambda_{k}(s-z)^{-1}=z^{k+2}$, and the $k^{\text {th }}$ evolution in the KP hierarchy which corresponds to $\dot{T}=\left[z^{k+2}, T\right]$, may be written as

$$
\dot{T}=\lambda_{k}\left[(s-z)^{-1}, T\right] \text {. }
$$

Since $\lambda_{k}$ is linear and commutes with everything in sight, it provides another way of writing the corresponding evolution of $u$ :

$$
\lambda_{k} \dot{u}=\lambda_{k} \partial_{1}(\tilde{m}(x, s) m(x, s))
$$

But both $\tilde{m}$ and $m$ can be expressed as asymptotic series in $s^{-1}$ with coefficients in the ring of smooth functions, up to order $k+2$ if $T$ has sufficient decay. The coefficients can be determined recursively by the relations in (V.14): 


$$
\begin{aligned}
& m(x, s)=\sum_{n=0}^{\infty} \frac{M_{n}(x)}{s^{n}}, \quad M_{0}(x) \equiv 1, \quad 2 \partial_{1} M_{n+1}(x)=\left[u(x)-\partial_{1}^{2}+\partial_{2}\right] M_{n}(x) . \\
& \tilde{m}(x, s) \sum_{n=0}^{\infty} \frac{\tilde{M}_{n}(x)}{s^{n}}, \quad \tilde{M}_{0}(x) \equiv 1, \quad 2 \partial_{1} \tilde{M}_{n+1}(x)=\left[-u(x)+\partial_{1}^{2}+\partial_{2}\right] \tilde{M}_{n}(x) .
\end{aligned}
$$

Writing down the formula for the $k^{\text {th }}$ evolution in the KP hierarchy amounts to solving for $M_{n}, \tilde{M}_{n}, n \leqq k+3$ in terms of $u(x)$, multiplying together the two series for $m, \tilde{m}$, and then picking out the coefficient of $s^{-k-3}$.

Because of the boundedness of the evolution (V.14), one obtains a global existence theorem for the whole KP hierarchy:

Theorem V.3. Suppose $u_{0}(x)$ has small derivatives up to order $k+7$ in $L^{1} \cap L^{2}\left(\mathbb{R}^{2}\right)$. Then the $k^{\text {th }}$ evolution equation in the KP hierarchy has a solution $u(x, t)$ for all $t \geqq 0$ with $u(x, 0)=u_{0}(x)$ and $u$ is uniformly bounded for all t in the space of $L^{2}$ functions with bounded Fourier transform.

Proof. Count the derivatives of $u$ needed to insure the continuity of all maps.

\section{References}

[ABF] Ablowitz, M. J., Bar Yaacov, D., Fokas, A. S.: On the inverse scattering transform for the Kadomtsev-Petviashvili equation. Stud. Appl. Math. 69, 135-143 (1983)

[AKNS] Ablowitz, M. J., Kaup, D. J., Newell, A. C., Segur, H.: The inverse scattering transformFourier analysis for nonlinear problems. Stud. Appl. Math. 53, 249-315 (1974)

[BC] Beals, R., Coifman, R. R.: Scattering, transformations spectrales, et equations d'evolution nonlineaire I, II. Seminaire Goulaouic-Meyer-Schwartz 1980/1981, exp. XXII and 1981/1982, exp. XXI, École Polytechnique, Palaiseau

[BC2] Beals, R., Coifman, R. R.: Multidimensional inverse scattering and nonlinear PDE. AMS Proc. Symp. Pure Math. 43 (1985)

[DJKM] Date, E., Jimbo, M., Kashiwara, M., Miwa, T.: Solitons,' $\tau$-functions, and Euclidean Lie algebras. RIMS Preprint, Kyoto 1981

[F] Frenkel, I. B.: Representations of affine Lie algebras, Hecke modular forms, and Korteweg-de Vries type equations. Yale preprint, New Haven 1982

[FA] Fokas, A. S., Ablowitz, M. J.: On the inverse scattering and direct linearizing transforms for the Kadomtsev-Petviashvili equation. Phys. Lett. A94, 67-70 (1983)

[K] Konopelchenko, B. G.: General structure of nonlinear evolution equations in $1+2$ dimensions integrable by two-dimensional Gelfand-Dickey-Zakharov-Shabat spectral problem and their transformation properties. Commun. Math. Phys. 88, 531-549 (1983)

[KP] Kadomtsev, B. B., Petviashvili, V. I.: On the stability of solitary waves in weakly dispersing media. Sov. Phys., Dokl. 15, 539 (1970)

[M] Manakov, S. V.: The inverse scattering transform for the time-dependent Schrödinger equation and Kadomtsev-Petviashvili equation. Physica 3D, 420-427 (1981). See especially p. 422.

Communicated by C. H. Taubes

Received June 9, 1986 
